\title{
Reconstrucción del aparato extensor de la mano a través de la cobertura cutánea. A propósito de un caso
}

\author{
J. Delgado Martínez ${ }^{(1)}$, J. Rodrigo Palacios ${ }^{(1)}$, E. Monclús Fuertes ${ }^{(2)}$, \\ E. González Peirona ${ }^{(3)}$ \\ Servicio de Cirugía Plástica y Reconstructiva del Hospital Universitario Miguel Servet de Zaragoza \\ (1) MEDICO ADJUNTO \\ (2) MÉdico RESIDENTE \\ (3) JEFE DE SERVICIO
}

Correspondencia:

Dr. Julio Delgado Martínez

Hospital Universitario Miguel Servet

$\mathrm{P}^{\mathrm{o}}$ Isabel la Católica, 1-3

50006 Zaragoza

Teléfono:976765500

e-mail: juldelgado@yahoo.com

\begin{abstract}
Los traumatismos graves sobre el dorso de la mano suelen comprometer el aparato extensor y provocan adherencias cicatriciales entre las diferentes estructuras afectadas que se traducen en rigidez. Presentamos un caso de cobertura y reconstrucción tendinosa de la mano utilizando el tejido subcutáneo del colgajo transferido como superficie de deslizamiento de aparato extensor.
\end{abstract}

Palabras clave: reconstrucción tendones extensores, cobertura del dorso de la mano,
Serious dorsal hand traumatisms usually affect the extensor system producing adhesions between the different involved structures resulting in stiffness. We present a case of dorsal hand coverage and complete tendinous reconstruction passing through the subcutaneous of the transferred free flap to create a gliding surface.

Key words: extensor tendon reconstruction, dorsal hand coverage,

Rev. Iberam. Cir. Mano - Vol. 37 • Núm. 1 •Mayo 2009 (58-61)

\section{INTRODUCCIÓN}

$\mathrm{E}$ 1 aparato extensor es muy vulnerable a nivel del dorso de la mano debido a su delgadez por lo que cualquier traumatismo puede afectarlos. Las lesiones complejas a este nivel que presentan pérdida de sustancia cutánea, lesión del aparto extensor y de las partes musculoesqueléticas subyacentes conducen a la formación de un lecho cicatricial en el que las adherencias tendinosas y la rigidez son la norma.

Existen varias técnicas para reconstruir la superficie de deslizamiento de los tendones y solu- cionar la cobertura al mismo tiempo. Exponemos un caso en el que solventamos estos problemas con un colgajo lateral de brazo parcialmente desgrasado e injertos tendinosos.

\section{CASO CLÍNICO}

Mujer de 48 años de edad que presenta politraumatismo con lesiones graves en cuero cabelludo y en el dorso de la mano izquierda. El equipo de urgencia realizó limpieza de las herida 


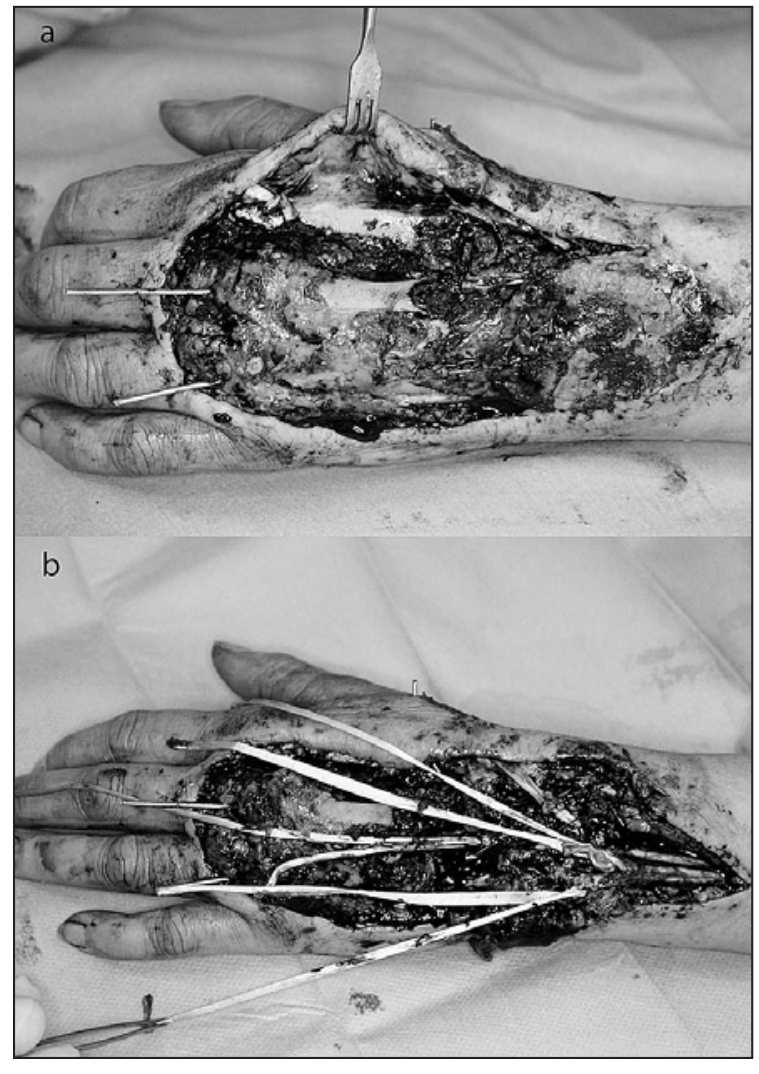

Figura 1. A: Defecto cutáneo, tendinoso y de partes blandas observado tras el desbridamiento. B: Tenorrafia proximal de los injertos tendinosos para reconstruir el ED.

apreciándose pérdida cutánea del dorso de la mano, pérdida de sustancia del extensor digitorum (ED) del $2^{\circ} 3^{\text {er }}$ y $4^{\circ}$ dedos, sección en su inserción del extensor carpi radialis brevis (ECRB) y longus (ECRL) y sección del extensor pollicis longus (EPL). Además, presentaba abrasión de la aponeurosis de la musculatura interósea dorsal y del dorso de los metacarpianos así como luxación carpometacarpiana. Se practicó de urgencia reducción y fijación con agujas de Kirschner de la luxación carpometacarpiana y cobertura con un colgajo interóseo posterior fascial injertado. La paciente precisó ingreso en UCI por las lesiones asociadas

En el postoperatorio se apreció necrosis distal del colgajo, quedando pendiente la reconstrucción del aparato extensor.

A los 8 dias, una vez pasado el periodo crítico, se reintervino a la paciente bajo anestesia general, se retiró el resto de colgajo interóseo posterior que estaba parcialmente necrosado (Fi-

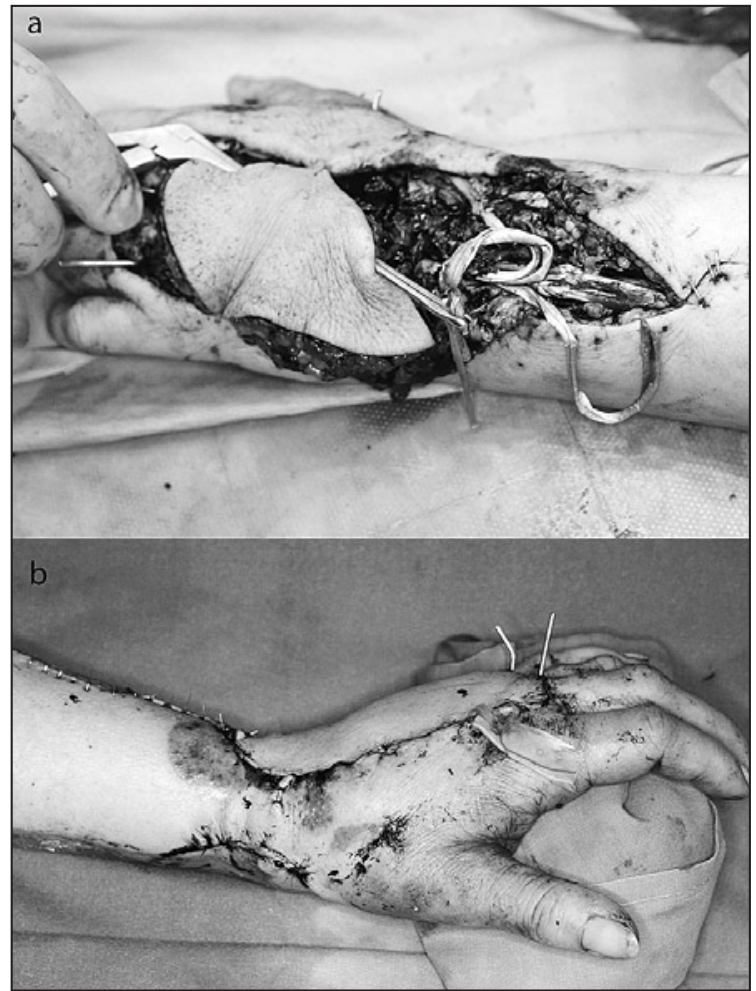

Figura 2. A: Paso de los injertos tendinosos a través de los canales subcutáneos. B: Resultado posquirúrgico.

gura 1A) y reparamos los tendones del ECRB y ECRL mediante reinserción en la base de los metacarpianos y el EPL mediante sutura directa, se reparó el retináculo extensor y obtuvimos injertos tendinosos procedentes de ambos plantaris y del extensor digitorum del $3^{\text {er }}$ dedo del pie para la reconstrucción del ED (Figura 1B). La cobertura cutánea se consiguió con un colgajo lateral de brazo parcialmente desgrasado en el que se realizaron varios canales subcutáneos por los que se pasaron los injertos tendinosos (Figura 2A). Tras realizar las tenorrafias, se completaron las anastomosis vasculares del colgajo a la arteria radial y a la vena cefálica. El tiempo quirúrgico fue de 6 horas (Figura 2B).

Tras 4 semanas de inmovilización se retiraron las agujas de Kirschner y se inició la rehabilitación, obteniendo a los 6 meses un rango de movimiento del carpo de $55^{\circ}$ y de $30^{\circ}$ para las metacarpofalángicas, con una limitación más acusada para la flexión y extensión casi completa.

Como consecuencia de la rigidez para la flexión pasiva que presentaban las articulaciones 


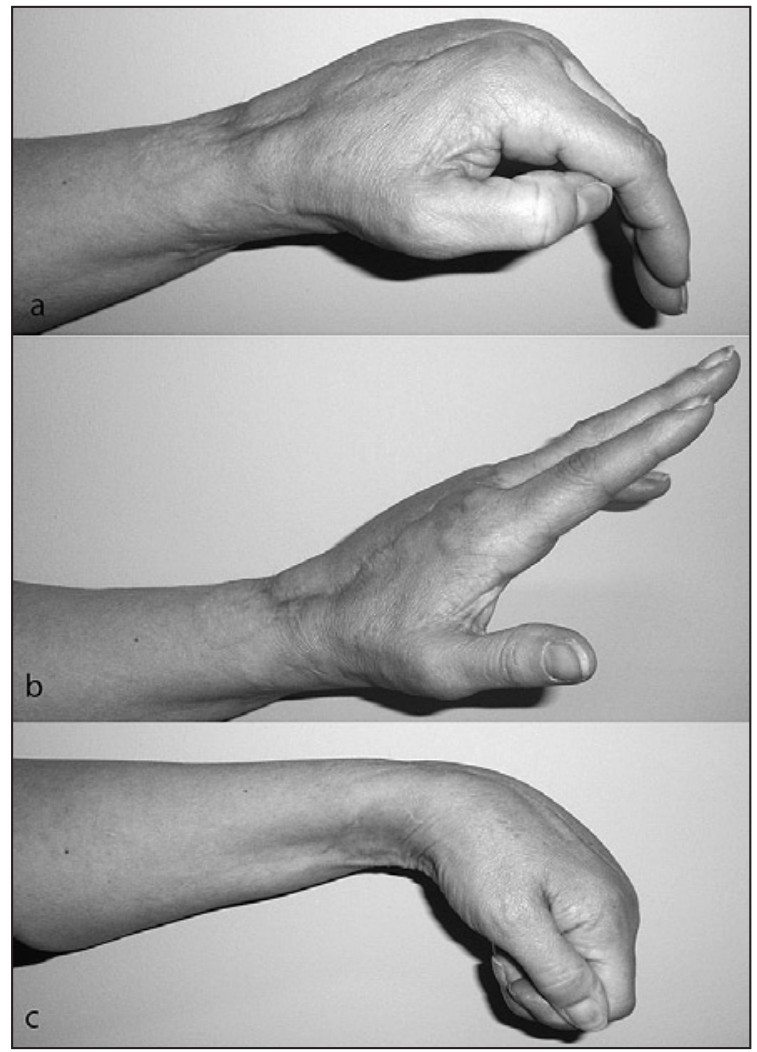

Figura 3. A: Rango de movimiento de las MCF en flexión. B: Rango de movimiento en extensión. C: Rango de movimiento del carpo en flexión.

metacarpofalángicas se decidió a los 10 meses realizar capsulotomía y tenolisis del aparto extensor mejorando el rango de movimiento que a los 15 meses era de $100^{\circ}$ en el carpo $65^{\circ}$ en la metacarpofalángica del $2^{\circ}$ dedo, $70^{\circ}$ en el $3^{\text {er }}, 50^{\circ}$ en el $4^{\circ}$ y $45^{\circ}$ en el $5^{\circ}$ (Figura 3 ).

\section{DISCUSIÓN}

Los traumatismos que producen pérdidas de cobertura en el dorso de la mano y lesionan, al mismo tiempo, el aparato extensor suelen provocar graves secuelas en la mano limitando de forma grave el rango de movimiento.

La necesidad de reparar o interponer injertos tendinosos, en ausencia del aparato extensor, entre la cobertura cutánea por un lado y el lecho cruento lesional formado por la musculatura interósea y los metacarpianos por otro, provoca adherencias entre los diferentes planos que limitan el rango de movimiento de los dedos.
Para minimizar las secuelas precisamos restaurar la cobertura, reconstruir el aparato tendinoso y crear una nueva superficie de deslizamiento para este. Aunque la estructura anatómica de mayor similitud con el dorso de la mano corresponde al dorso del pie, su transferencia microquirúrgica con el aparato tendinoso provoca secuelas en la zona donante que desaconsejan su uso ${ }^{1}$.

Otra opción reconstructiva en estas situaciones consiste en crear una cobertura con dos capas para aislar los injertos tendinosos en un ambiente adecuado para limitar las adherencias y que permitan un buen plano de deslizamiento. En este sentido, los colgajos adipofasciales injertados como el colgajo de fascia del músculo temporoparietalis o el de serratus, entre otros, permiten envolver el aparato tendinoso reconstruido $^{2,3}$. De igual forma pueden utilizarse colgajos fasciales con islas cutáneas que proporcionan una cobertura más elástica como el anterolateral de muslo cuyo componente adipofascial proporciona el plano de deslizamiento a los tendones ${ }^{4}$.

El problema principal que presentan los colgajos fasciocutáneos es el excesivo grosor, comparativamente hablando, que presentan en relación al espesor del dorso de la mano. En los últimos años se han difundido técnicas para adelgazar estos colgajos sin comprometer su vascularización lo que facilita su utilización en el dorso de la mano ${ }^{5}$.

En nuestro paciente usamos la opción reconstructiva consistente en utilizar un colgajo libre fasciocutáneo lateral de brazo $^{6}$, parcialmente desgrasado, para solucionar la cobertura y pasar los injertos tendinosos a través de canales creados en el tejido subcutáneo que actuará como superficie de deslizamient ${ }^{7}$.

Los resultados de esta técnica realizada en dos tiempos, tras interponer en un primer tiempo varillas de silicona y posteriormente realizar los injertos tendinosos, no han demostrado mejores resultados por lo que es preferible realizar la reconstrucción en un tiempo durante la fase aguda ${ }^{8}$.

En nuestra opinión esta técnica debe tenerse en cuenta en lesiones complejas del dorso de la mano, proporciona buena cobertura tras el desgrasado y un buen plano de deslizamiento para los tendones obteniéndose buenos resultados al igual que presentan el resto de técnicas que utilizan exclusivamente colgajos adipofasciales injertados. 


\section{BIBLIOGRAFÍA}

1 Samson M, Morris S, Tweed A. Dorsalis pedis flap donor site: aceptable or not? Plast Reconstr Surg, 1998; 102: 1549-54.

2 Biswas G, Lohani I, Chari P. The sandwich temporoparietal free fascial for tendon gliding. Plast Reconstr Surg, 2001; 108: 1639-45.

3 Schwabegger A, Hussl H, Rainer $\mathrm{C}$, et al. Clinical experience and indications of free serratus fascia flap: A report of 21 cases. Plast Reconstr Surg, 1998; 102: 1939-46.
4 Muneuchi G, Suzuki S, Ito O, et al. Free anterolateral thigh fasciocutaneous flap with a fat/ fascia extension for reconstruction of tendon gliding surface in severe bursitis of the dorsal hand. Ann Plast Surg, 2002; 49: 312-6.

5 Adani R, Tarallo L, Marcoccio I, et al. Hand reconstruction using the thin anterolateral thigh flap. Plast Reconstr Surg, 2005; 116: 467-73.

6 Katsaros J, Schusterman M, Beppu M, et al. The lateral upper arm flap. Anatomy and clinical applications. Ann Plast Surg ,1984; 12: 489-500.

7 Scheker L, Langley S, Martin D, et al. Primary extensor tendon reconstruction in dorsal hand defects requiring free flaps. J Hand Surg Br, 1993; 18: 568-75

8 Sumdine M, Scheker L. A comparison of inmediate and staged reconstruction of the dorsum of the hand. J Hand Surg, 1996; 21B: 216-21. 